\title{
A Performance Survey on Deflection Routing Techniques for OBS Networks
}

\author{
Oscar Pedrola ${ }^{1}$, Sébastien Rumley ${ }^{2}$, Davide Careglio ${ }^{1}$, Miroslaw Klinkowski ${ }^{1,3}$, Pedro Pedroso $^{1}$ \\ Josep Solé-Pareta ${ }^{1}$, Christian Gaumier ${ }^{2}$ \\ ${ }^{1}$ CCABA, Universitat Politècnica de Catalunya, 08034 Barcelona, Spain \\ ${ }^{2}$ Swiss Federal Institute of Technology (EPFL), CH-1015 Lausanne, Switzerland \\ ${ }^{3}$ National Institute of Telecommunications (NIT), 1 Szachowa Street, 04-894 Warsaw, Poland \\ Tel: (+34) 93 4016985, Fax: (+34) 93401 7055, e-mail: careglio@ac.upc.edu
}

\begin{abstract}
In this paper, we present a survey comparing different deflection routing based techniques applied to optical burst switching (OBS) networks. For such study we consider the E-OBS architecture proposed in [1] which is an advantageous solution for OBS networks since routing decision can be taken freely inside the network without constraints on the length of the path. Under this environment, several effective routing strategies proposed in the literature are applied, namely deflection routing, reflection routing, reflection-deflection routing and multitopology routing. The aim of this study is to analyse all these techniques considering both asynchronous and synchronous burst arrivals and compare their benefits. Moreover, we focus on a quasi-synchronous burst arrival case (with bursts not perfectly aligned) and analyse the trade-off between performance and alignment.
\end{abstract}

Keywords: optical burst switching, routing algorithm, performance analysis.

\section{INTRODUCTION AND MOTIVATION}

Two main features distinguish Optical Burst Switching (OBS) [2] from other optical switching technologies: the transmission of large data bursts, which are aggregated at the edge of the network, and the possibility to establish a path dynamically and on-the-fly (i.e. without acknowledgment of the availability of transmission resources). Because of the absence of optical buffering capabilities, the main challenge of OBS networks is to deal with high burst losses that arise due to the contention of bursts transmitted in the network.

To mitigate the burst contention problem there have been proposed solutions based on deflection (or alternative) routing. All these methods allow re-routing contending bursts from primary to alternative routes and, by these means, alleviating congestion on bottleneck links and achieving dynamic load balancing in the network. In this paper we focus on the so-called offset time-Emulated OBS (E-OBS) network architecture [1] which facilitates the application of alternative routing since routing decision can be taken freely inside the network without constraints on the length of routing path.

In principle the transmission of optical bursts is asynchronous in an OBS network. That means that bursts are not aligned each other and they arrive at a core switching node in casual instances of time. Performance improvements can be achieved if synchronous operation is applied: in fact, in such a case contention may occur only between entire data units and better transmission resource utilization can be obtained with simple contention resolution mechanisms [3]. Such synchronous operation was proposed in the past to the optical packet switching (OPS) networks (see e.g. [4]), however synchronisation blocks are bulky and complex and they have not been considered widely in OBS networks.

In this paper we focus on a quasi-synchronous operation in OBS networks. In such a scenario, we do not include any synchronisation blocks in the network; on the contrary, we assume that the network links are designed such that the resulting propagation delay corresponds to a multiple of a given fixed time slot duration and the bursts are released only at the beginning of time slots. To take into account that perfect synchronisation is practically impossible, we accept the presence of some skew of the clocks at the edge nodes (for that we refer to quasi-synchronous operation) and hence the bursts arriving at core nodes are not perfectly aligned.

Summarizing, the goal of this paper is twofold. First, we focus on several effective deflection routing techniques proposed in the literature [5]-[9], and present a comparative performance study that is conducted in an asynchronous and synchronous E-OBS network scenario. Then, we analyse if the quasi-synchronous network operation can have any benefits in terms of performance. If this is a case we can motivate further study on how to achieve such quasi-synchronous burst arrivals.

The paper is organised as follows. In Section 2 we present some details of analysed network scenarios. In Section 3 we describe briefly deflection routing techniques that we consider. In Section 4 we report on simulation results. Finally, in Section 5 we conclude the study.

\section{OBS NETWORK SCENARIOS}

In this study we consider an E-OBS network scenario [1]. In particular, core switching nodes are enhanced with a pool of fibre delay coils that is inserted into the data path at the input port of the node. On the contrary to conventional OBS (C-OBS) architectures where the processing offset time is provided at the edge node by 
delaying the transmission of a burst with respect to its control packet, in E-OBS the offset times are provided by means of the fibre delay coils at each core node. By that means, both the burst and control packet can be sent together from the edge node, thus avoiding several problems that result from the offset time variation inside the network, a feature that is inherent to C-OBS. For instance, concerning routing management, it is advantageous to provide offset times at each core node since routing decisions can be taken freely inside the network without constraints on the length of the path. Contrarily, in C-OBS the maximal length of routing path is related to the offset which, once introduced at the edge node, decreases at each hop.

In general the transmission of optical data in the network can be either asynchronous or synchronous. Although both approaches have been studied extensively in the context of OPS networks, still the research on OBS concerns mostly the asynchronous approach.

In asynchronous OBS networks, optical bursts are released from edge nodes at arbitrary (random) instances of time and they are not aligned when they arrive at core switching nodes (see Figure 1A). Accordingly, the switching operation is performed asynchronously. The advantage of this approach is the simplification of the burstification process and the low complexity of switching nodes.

The idea of synchronous, or time-slotted, transmission in optical networks has been considered mainly in the context of OPS networks. In case of synchronous OBS scenario, optical bursts are aligned and transmitted synchronously at the beginning of a fixed-duration time slot (see Figure 1B). The main advantage of the synchronous approach, with respect to the asynchronous approach, is the improvement of the overall burst loss performance. In synchronous OBS networks, as far as bursts travelling over different length links may still arrive at a core node in different instances of time, their synchronization is achieved by a specialised node input interface. Since such device is very complex, the synchronous case is usually not considered a viable solution.

Nonetheless in this paper we consider both asynchronous and synchronous cases and use them as benchmarking references. The main aim is to analyse the quasi-synchronous network operation. In such scenario, there are no synchronisation devices; the network links are designed so that their length would correspond to a multiple of the slot size and the edge nodes are synchronised each other and release bursts at the beginning of time slots. Since there is difficult to have a perfect synchronisation at the edge nodes, so that all had the same clock information, we assume that there is some skew of the clocks at the edge nodes and hence the bursts arriving at core nodes are not perfectly aligned (see Figure 1C) with a consequence of having performance degradation compared to the perfectly synchronous case.

To prevent the overlapping of de-synchronised bursts, we consider that the bursts do not occupy the slots completely but there is some guard band introduced between them. This guard-time should be large enough to maintain the burst alignment as in the case of synchronous operation but not too much in order to not decrease excessively the bandwidth utilisation. As a consequence, the duration of the guard-time results in a clear tradeoff between performance and alignment.

Note that on the contrary to OPS networks where optical packets have small size and even their small misalignment might result in a serious degradation of performance, in OBS there is possibly a higher margin for de-synchronization of burst transmission due to the much larger burst durations and, as a result, much larger guard bands. For these reasons, we expect that the results obtained with the quasi-synchronous operation should be somewhere between asynchronous and synchronous cases.

In Section 4 we study the impact of the clock skew on the burst loss performance in the quasi-synchronous OBS network. The question of how to maintain the quasi-synchronisation at the edge nodes is out of the scope of this paper and is left for further study.

A. Asynchronous

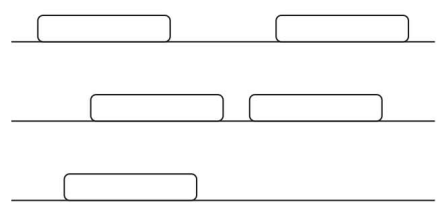

B. Synchronous

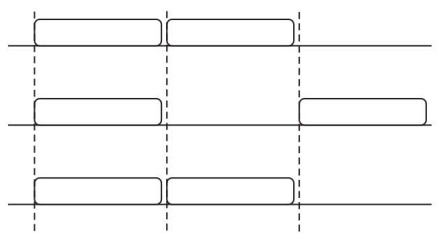

C. Quasi-synchronous

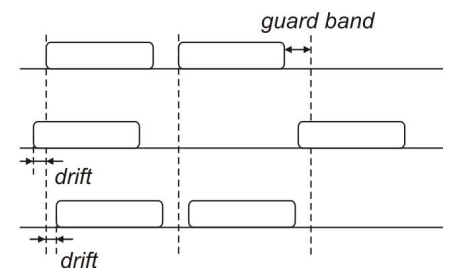

Figure 1. Asynchronous vs. synchronous vs. quasi-synchronous burst transmission.

\section{DEFLECTION ROUTING ALGORITHMS}

All the analysed deflection algorithms perform hop-by-hop routing, i.e., the routing decision is taken at each intermediate node and no paths are pre-established in the network. In particular, the routing decision only takes into account the selection of the next node and is based on the information stored in routing tables. The routing tables are built with the assistance of the Dijkstra's shortest path algorithm which finds the shortest routes with respect to the number of hops. Both the tables of primary routes and alternative routes are built. If there is a burst contention at the output port of a primary route, an alternative route is considered. The selection of the 
alternative route depends on the particular technique implemented in the routing algorithm. In order to avoid excessive burst circulations in the network we consider the use of a time-to-live (TTL) field in the burst control packet (BCP), which limits the number of hops a burst can undertake.

Concretely, our study concerns five different deflection routing strategies that we briefly describe below. For more details concerning the algorithms, readers are referred to the corresponding references.

\subsection{Deflection Routing (DR)}

This is the simplest version of the deflection routing algorithm [5] where in case of burst contention in the primary output port, an alternative one, if not occupied, is selected at the switching node. In our implementation of the algorithm there is only one alternative route for each destination at each node, and this route is the second shortest path route. To save resources in the network, bursts are deflected only if they have enough TTL to reach the destination through the alternative route. If the TTL is not large enough, the burst is simply discarded. Note that the first shortest path route is the primary route.

\subsection{Reflection Routing (RR)}

The idea of reflection routing comes from OPS networks [6]. A reflection routing algorithm allows sending a contending burst towards a neighbour node (reflection neighbour) on the condition that this reflection neighbour, after receiving the burst, will intent to return the burst back or, in other words, reflect it. The idea behind this mechanism is to use network links as virtual fibre delay buffers with the expectation to be able to re-forward the contending burst towards its destination after a period of time, which corresponds to the propagation delay on the reflective links.

In our implementation of the classic reflection routing algorithm, reflection neighbour nodes are selected with a first-fit policy and amongst all the output ports with available resources. Moreover, if the burst TTL is not large enough to support the reflection, which would add two supplementary hops before reaching the destination, the burst is dropped.

\subsection{Load Balanced Reflection Routing (LBRR)}

This algorithm is a slightly modified version of the classic RR algorithm adapted to OBS networks presented in [7]. The extension concerns the selection method of the neighbour node at which the reflection is performed. In this proposal, the traffic coming from neighbour nodes is monitored so that the node of the lowest load can be distinguished. As far as such node has the most chances to reflect a burst back it is selected by the reflection algorithm. Here, in the same way as in the classic RR algorithm, the TTL is checked before the reflection is done.

\subsection{Reflection-Deflection Routing (RDR)}

The idea behind this algorithm is the concatenation of both the RR and DR algorithms [8]. In this approach when burst contention occurs, the reflection algorithm is started. If the reflection to a neighbour node is successful, the returning burst may still find resources in the primary output port occupied. In such case, using either classic RR or LBRR, the burst would be just discarded. Conversely, RDR allows the burst to be deflected through an alternate output port, which corresponds to the second shortest path route.

Our implementation makes use of the RR and DR policies previously described.

\subsection{Multi-Topology Routing (MTR)}

A multi-topology deflection routing algorithm was proposed so that to avoid excessive burst circulations in the network without the need for an additional TTL indicator [9]. The main idea is to build primary and alternative routing tables that guarantee loop-free forwarding inside the network. Then, each alternative routing table is assigned a numeric index. Once contention occurs, a burst can only be deflected by using information stored in a routing table with higher index than the current one. In this way, the number of deflections a burst can undergo is limited to the number of alternative routing tables.

\section{SIMULATION RESULTS}

\subsection{Scenario}

To obtain the results, we have carried out simulations using the JAVOBS network simulator [10] which implements an OBS discrete event simulation model. All simulations were run under the following assumptions: (1) 16 bidirectional wavelengths at $10 \mathrm{Gbps}$ per link; (2) equal link lengths set to $200 \mathrm{~km}$; (3) traffic is uniformly distributed; (4) the overall network load is set to half of the network capacity; (5) nodes equipped with full wavelength conversion; (6) the switching and processing time are neglected for sake of simplicity.

For all cases we consider fixed burst length size set to $30 \mu \mathrm{s}$. For the asynchronous case, exponential burst interarrival time distribution is assumed while, for the synchronous and quasi-synchronous cases, the bursts can only depart at the beginning of the slot. Note that for the quasi-synchronous case, the burst can depart with some skew with respect to the beginning of the slot. 


\subsection{Comparative results}

Figure 2 shows the relative gain comparing the asynchronous and synchronous cases adopting the five different deflection routing algorithms described in Section 3. Giving the burst loss probability of the asynchronous case $\left(B L P_{\text {asyn }}\right)$ and the one of the synchronous case $\left(B L P_{\text {syn }}\right)$, we define the relative gain as $\left(B L P_{a s y n}-B L P_{\text {syn }}\right) /$ $B L P_{a s y n}$. For this comparison we randomly generate 10 different topologies of 20 nodes (each one having a degree distribution following a power law) with different clustering coefficients and nodal degrees. Each rhombus marker in the figures represents the relative gain obtained in one topology. Since each topology has a different clustering coefficient and nodal degree, we depict the trendline in the figure.

We can observe that all algorithms have the same trend: the relative gain slightly decreases with the increase of the clustering coefficient while significantly increases with the increase of the nodal degree.

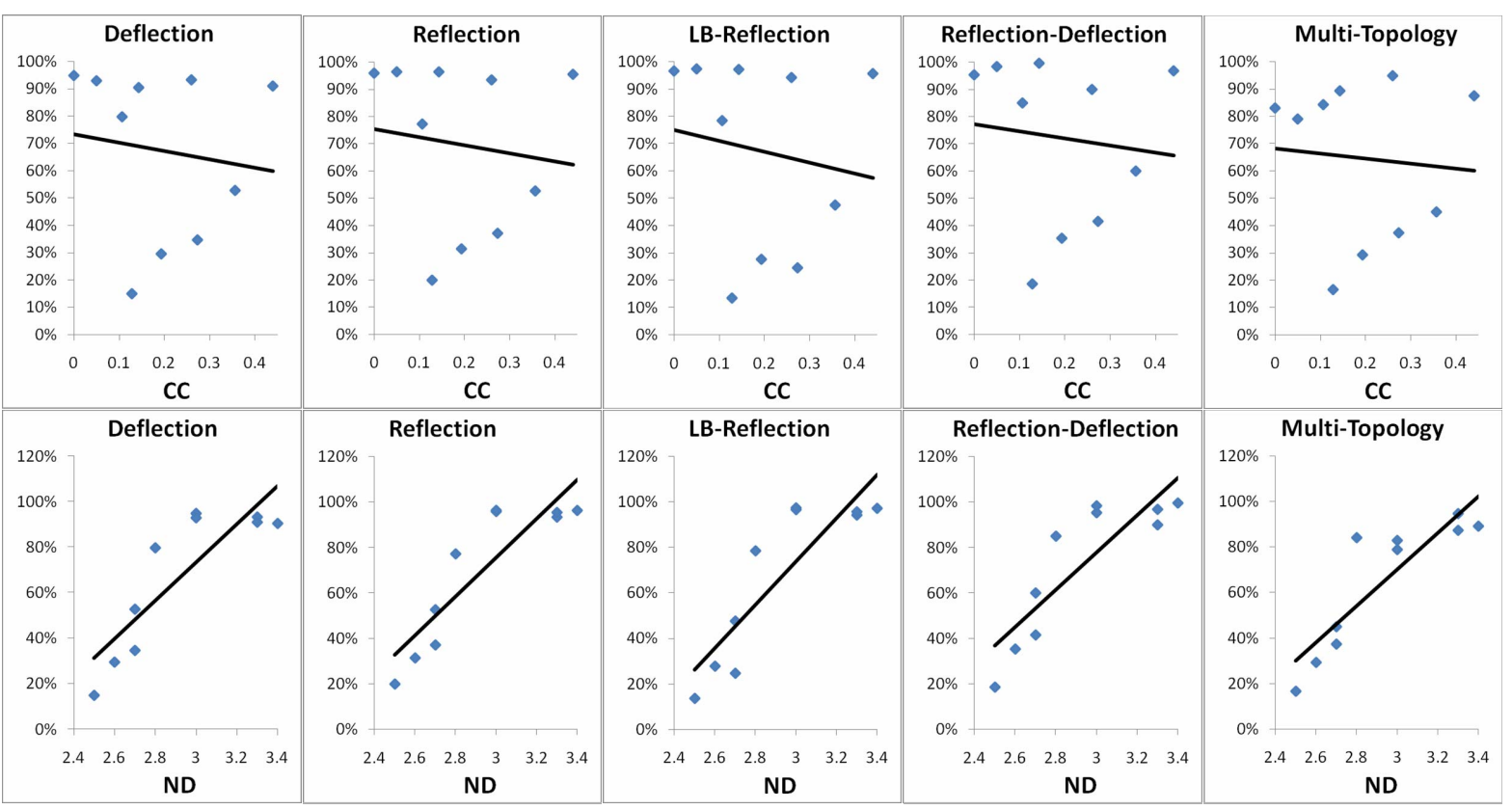

Figure 2. Relative gain as a function of the clustering coefficient (CC) and nodal degree (ND).

Figure 3 compares the deflection routing algorithms using the topology with the higher nodal degree value. As we can observe in the figure, the reflection-deflection routing (RDR) obtains the lower burst loss probability under the synchronous operation. On the contrary, all algorithms behave similar under the asynchronous operation.

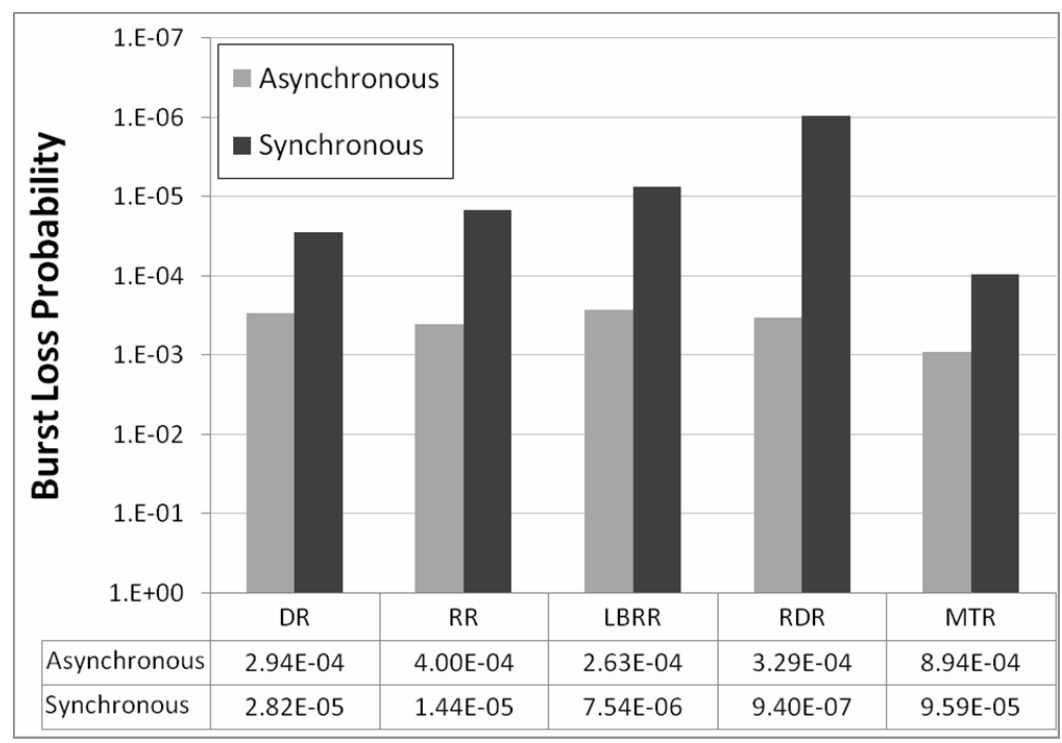

Figure 3. Performance comparison between deflection routing algorithms.

\subsection{Quasi-synchronous results}

For the following study we only consider the reflection-deflection routing algorithm and the random topology with the higher nodal degree. 
For the quasi-synchronous case, we consider that each node introduces a skew in the burst departure time. This skew follows a Gaussian distribution with mean set to zero and a given variance (sigma). We suppose that this variance is known and the guard-time between bursts is set according to its value. We consider three possible cases: 1) a guard-time equal to the variance (1-sigma) - since the skew is Gaussian distributed there will be $68.2 \%$ of burst departures occurring during such guard time; 2 ) a guard time equal to two times the variance (2-sigma, 95.4\%); 3) a guard time equal to three times the variance (3-sigma, 99.6\%).

Figure 4 shows the burst loss probability as a function of the variance of the skew, which is at the same time a percentage of the slot time. As a reference we include the asynchronous and synchronous results. We can observe that with lower skews, the curves of 1-sigma, 2-sigma and 3-sigma are overlapped. As the skew increases, best results are obtained using only 1-sigma of guard-time. It is important to notice that with relatively high values of the skew, the burst loss probability is still under the ones obtained with the asynchronous case.

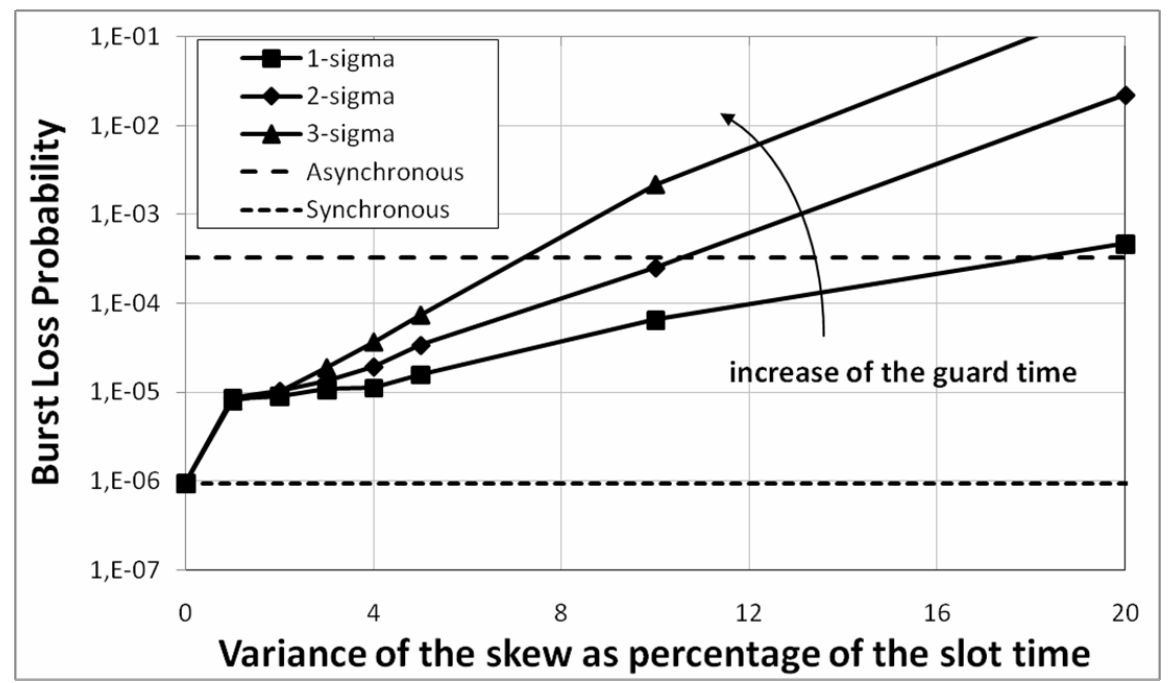

Figure 4. Burst Loss Probability as a function of the variance of the clock skew (in percentage).

\section{CONCLUSIONS}

In this paper we have compared five different deflection routing algorithms considering both synchronous and asynchronous burst interarrival times. This comparison has been performed using 10 different topologies of 20 nodes. The obtained results highlight that the relative gain of the synchronous case over the asynchronous one is dependent of the nodal degree of the network. Between the algorithms, the reflection-deflection routing is the one with the lower burst loss probability.

Moreover, an important outcome of this study is the introduction of the quasi-synchronous operation. In fact, since obtaining a perfect synchronisation is difficult, in such mode of operation we allowed some degree of de-synchronisation between the clocks of the nodes. The results indicate that significant improvements (more than one order of magnitude for the clock skew under approximately 8\%) can be obtained with respect to the pure asynchronous case and motivate further investigation on the quasi-synchronous operation in OBS networks.

\section{ACKNOWLEDGEMENTS}

This work was partially funded by the European Commission under the BONE (Building the Future Optical Network in Europe) Network of Excellence of the 7th ICT Framework Program.

\section{REFERENCES}

[1] M. Klinkowski, D. Careglio, J. Solé-Pareta, and M. Marciniak, "Performance overview of the offset time emulated OBS network architecture", to appear in IEEE/OSA J. Lightwave Technol., 2009.

[2] C. Qiao and M. Yoo, "Optical burst switching (OBS) - a new paradigm for an optical Internet", J. High Speed Netw., vol. 8, pp. 69-84, 1999.

[3] F. Kuo, The ALOHA system, Computer Networks, Prentice-Hall, pp. 501-518, 1973.

[4] C. Guillemot et al., "Transparent optical packet switching: the European ACTS KEOPS project approach", IEEE/OSA J. Lightwave Technol., vol. 16, no. 12, pp. 2117- 2134, Dec. 1998.

[5] C. Hsu, T. Liu, and N. Huang, "Performance analysis of deflection routing in optical burst-switched networks", IEEE INFOCOM 2002, New York, USA, Jun. 2002.

[6] H. Yokoyama and H. Nakamura, "Mechanisms and performance of reflection routing for optical packet switched networks", OSA OFC 2002, pp.779-781, 2002. 
[7] J. Perelló, S. Spadaro, J. Comellas, and G. Junyent, “A Load-Based Reflection Routing Protocol with Resource Pre-Allocation for Contention Resolution in OBS Networks”, Eur. Trans. Telecomms., no. 20, pp. 1-7, 2009.

[8] M. Morita, H. Tode, and K. Murakami, "Reflection-Based Deflection Routing in OPS Networks", IEICE Trans. Commun., vol. E91-B, no. 2, pp. 409-417, 2008.

[9] S. Gjessing, “A Novel Method for Re-Routing in OBS Networks", IEEE ISCIT 2007, pp. 22-27, New York, USA, Oct. 2007.

[10] O. Pedrola, S. Rumley, M. Klinkowski, D. Careglio, J. Solé-Pareta, and C. Gaumier, "JAVOBS: A Flexible Simulator for OBS Network Architectures", submitted to Journal of Networks, 2009. 